\title{
Urinary Incontinence and its impact on Quality of Life among Frail Elderly Males
}

\section{Nahla M. Zaki 1, Rania M. Abou-Hashem 1, Hala S. Sweed 1, Suzan Mounir Ali 1}

${ }^{I}$ Geriatrics and Gerontology Department, Faculty of Medicine, Ain Shams University

\begin{abstract}
Background: Urinary incontinence (UI) is a major geriatric problem, that is underestimated in frail older males.
\end{abstract}

Aim: To identify risk factors of impaired QOL among frail older males with UI.

Methods: A cross-sectional study including 120 frail older males attending Geriatric Hospital at Ain Shams University Hospitals. Sixty subjects had UI. Participants were tested by the Arabic version of International Consultation on Incontinence Questionnaire-Urinary Incontinence Short Form (ICIQ-UI SF), the American Urological Association symptom index (AUA-SI) and the Arabic version of Incontinence Impact Questionnaire; short form (IIQ-7 SF) to measure the severity of $\mathrm{UI}$, lower urinary tract symptoms and health-related $\mathrm{QOL}$ respectively.

Results: Mixed form of UI (40\%) was the most prevalent type followed by urge UI (38.3\%). Prevalence of depression was $56.67 \%$. More than $90 \%$ expressed emotional health affection and more than two thirds had difficulty praying, travelling and performing physical and social activities. Most of the domains were affected in patients with mixed or urge urinary incontinence, compared with other types of UI. A positive relationship was found between severity of UI symptoms and severity of QOL impairment in all domains. UI-associated QOL was positively affected by social isolation, depression, functional dependence, advanced frailty status, severe UI, long UI duration, presence of chronic constipation and using alpha-blockers. Social isolation was the only independent predictor for decreased UIassociated QOL.

Conclusion: $U \mathrm{Ul}$ exerts negative impact on $\mathrm{QOL}$ of frail older males through social and psychological factors, functional level, frailty status, comorbidity, medication use, and severity and duration of UI symptoms.

Keywords: urinary incontinence, frail older males, $Q O L$

\section{Background}

Urinary incontinence (UI) is one of the geriatric giants that affects $15-30 \%$ of community dwelling older adults and up to $80 \%$ of nursing homes residents (Prud'homme et al., 2018) [1].

Older population is a rapidly growing population that encompasses wide range of individuals varying in physical, functional and cognitive capabilities (Fonda et al., 2005) [2].

Frail elderly represent a clinical phenotype characterized by reduced physiological reserve and high vulnerability to morbidity and mortality with acute stressors (Kojima et al., 2019) [3]. They usually have multiple comorbidities and functional dependence (Fonda et al., 2005) [2].

UI is a common condition in frail older people due to the presence of multiple interacting factors that precipitate loss of continence and aggravate urinary symptoms (Wagg et al., 2014) [4].

The perspectives of frail older people regarding healthrelated QOL include physical (health) and psychological wellbeing, self-dependence and social interaction (Kwong et al., 2014) [5] (Puts et al.,2006) [6]. It was found that prevalent and incident UI exert negative influence on different domains of QOL in frail older individuals (DuBeau et al., 2006) [7]. This may support the concept which states that improving UI enhances health-related QOL and decreases adverse outcomes of UI as falls, pressure ulcers and urinary tract infections. Some authors consider that the use of disease specific tools to assess QOL is more sensitive than general tools to find out the aspects of this disease (Dugger, 2010) [8].

UI had long been studied in females, meanwhile it is a prevalent distressing problem among older males that increases with advancing age (Anger et al., 2006) [9]. Elderly males may have variable bothersome lower urinary tract symptoms (LUTS) including UI, which are all forms of bladder dysfunction (Griebling, 2008) [10]. In the current study, we are trying to focus on this understudied group i.e. frail elderly incontinent males and to analyze the means by which UI can affect health-related QOL by using a disease-specific tool in 
evaluating quality of life.

\section{Aim of the study:}

The aim of the study was to determine the impact of type, severity and duration of UI symptoms on different domains of QOL, and to define the predictors of impaired UI-associated QOL among frail older males in the Geriatric Medicine Hospital at Ain Shams University Hospitals.

\section{MATERIALS AND METHODS}

The current study is a cross-sectional study. A total of 120 frail elderly males attending Geriatric Hospital (outpatient clinics and inpatient department) at Ain Shams University Hospitals were examined along a period of 6 months. Sample size was calculated using Pass program, setting the type- 1 error $(\alpha)$ at 0.05 and the confidence interval width at 0.1 . Result from previous study (Prudhomme et al., 2017) showed that $6.6 \%$ of males had a lifetime prevalence of Urinary incontinence. Calculation according to these values produced a sample size of 120 males, taking in account $20 \%$ drop out rate. Amongst them, 60 patients had UI symptoms and continued the assessment. Inclusion criteria were: frail males aged 60 years or more. We excluded patients with dementia, subjects who were unwilling to participate in the study, catheterized patients and non-frail patients. Assessment included the Mini-Mental State Examination; MMSE (Folstein et al., 1975) [12], Arabic version (Elokl et al., 2002) [13] for exclusion of cognitively impaired subjects.

Diagnosis of frailty was done using the clinical frailty scale (CFS) (Rockwood et al., 2005) [14]. The CFS ranges from 1 (very fit) to 9 (terminally ill) based on descriptors and pictographs of activity and functional status (Juma et al., 2016) [15]. Patients were classified into 3 categories: mild, moderate and severely frail.

Data collected from participants were: background characters as age, education level, special habits of medical importance such as smoking and alcohol intake. Comprehensive Geriatric Assessment was done, through a thorough medical history including comorbid conditions, medications review and assessment of UI regarding type, duration and severity. Baseline functional level was assessed by using activities of daily living (ADL) (Katz et al., 1963) [16] and instrumental activities of daily living (IADL) (Lawton and Brody, 1969).

Screening for depression was done through using the Arabic version (Shehata et al., 1998) [18] of geriatric depression scale (GDS-15) (Sheikh and Yesavage, 1986) [19]. The scale comprises 15 Yes/No questions, the subject is considered to be depressed if the final score is $\geq 5$.

The type of incontinence was determined according through answering some questions (Lagro-Janssenet al., 1995) [20]: "do you have a strong urge that leakage can occur on the way to toilet?, "does leakage occur at moments of increased pressure, for example, when sneezing or coughing?" and "does leakage of few drops occur all the time?". Accordingly, urge incontinence is the involuntary loss of urine associated with urgency. Stress incontinence is the involuntary loss of urine on physical effort or sneezing or coughing. Overflow incontinence is the loss of small amounts of urine in the symptomatic presence of over-distended bladder. Functional/disability associated incontinence is considered in subjects that have involuntary loss of urine due to functional inability to reach toilet in time due to physical or mental impairment (D'Ancona et al., 2019) [21]. Mixed incontinence, in our study, is the combination of two or more types of incontinence.

The Arabic version of International Consultation on Incontinence Questionnaire-Urinary Incontinence Short Form (ICIQ-UI SF) (Hashim et al., 2006) [22] is used in the assessment of the severity of UI. It comprises three-scored items to assess the frequency of urinary incontinence (score 0-5), the amount of urinary incontinence (score 0- 6), and its impact on the individual's QOL (score 0-10). There is an unscored self-diagnostic item to assess the perceived causes of leakage. The score is obtained by adding the scores from the three-scored items together, to give a score range between zero and 21 . The higher the score the greater the severity: mild (1-5), moderate (6-12), severe (13-18), very severe (19-21).

The American Urological Association symptom index (AUA-SI) was used to assess the severity of lower urinary tract symptoms (LUTS) symptoms. It includes 7 questions covering frequency, nocturia, weak urinary stream, hesitancy, intermittence, incomplete emptying and urgency (Barry et al., 1992) [23]. Every question measures the frequency of symptoms; none (0), less than one time in 5 (1), less than half the time (2), about half the time (3), more than half the time (4) or almost always (5). By adding scores of the 7 questions together the score ranges from 0 to 35 , categorized into: mild (score $0-7$ ), moderate (score 8-19) or severe (score 20-25).

The health-related QOL is measured by using the Arabic version of Incontinence Impact Questionnaire; short form (IIQ-7 SF) (El-Azab and Mascha, 2009) [24]. The (IIQ-7) questionnaire is a seven-item questionnaire designed to assess different domains of QOL impairment. The original IIQ-7 questionnaire consists of seven items covering four domains: physical activities, social relationships, travel, and emotional health. In Arabic version inquiring about prayer was added, inquiring about entertainment was deleted, and inquiring about social activities was modified to suit Egyptian culture. It has a four-point rating scale: $0=$ not at all, $1=$ slightly, $2=$ moderately, and $3=$ greatly; the higher the score the poorer QOL (El-Azab and Mascha, 2009) [24].

The Charlson comorbidity index (Charlson et al., 1987) [25] is used for assessing the burden of co- 
morbity. It consists of 17 comorbidity categories. Each comorbidity category is presented by a numerical weight based on relative 1-year mortality risk, and the sum of these weights gives a total comorbidity score (Quan et al., 2011) [26].

\section{Statistical analysis:}

The collected data were coded, tabulated, and statistically analyzed using IBM SPSS statistics (Statistical Package for Social Sciences) software version 22.0, IBM Corp., Chicago, USA, 2013. Quantitative date. e.g., age, weight, will be presented as mean and standard deviation.

Frequency and percentages are presented for all qualitative variables. Comparison between quantitative variables was done using t-test and comparison of qualitative variables was done using Chi square test. Correlations (r-value) were assessed by Spearman rank correlation to find relation between different variables. While positive $r$-value indicates direct correlation, negative $r$-value indicates inverse relationship between the variables. Significance level was determined according to $\mathrm{P}$ value (Probability): $\mathrm{P}>0.05$ insignificant, $\mathrm{P} \leq 0.05$ significant and $\mathrm{P}<0.01$ highly significant.

\section{Results}

The research population comprised 120 males meeting frailty criteria, $50 \%$ of them reported urinary incontinence, those represented our study group.

The basic characteristics of the study population are shown in Table (1). The participants had a mean age of 74.55 years, $53.33 \%$ were illiterate, $75 \%$ were smokers and $33.33 \%$ were obese. Most of them (80\%) were married. Regarding frailty status as assessed by CFS, $38.33 \%$ were mildly frail, $43.43 \%$ were moderately frail, and $18.33 \%$ were severely frail. By using GDS, $56.67 \%$ of participants were found depressed.

Table (2) shows the distribution of urinary incontinence types among the study population, the most prevalent type found was mixed UI (40\%) followed by urge UI $(38.3 \%)$.

Many domains of health-related QOL found to be affected in the study group, as shown in Table (3). Nearly $93 \%$ of incontinent subjects expressed emotional distress in the form of sense of depression and hopelessness, and $90 \%$ had sense of anxiety or frustration.

Regarding social activities, $86.6 \%$ experienced impairment of social participation and $80 \%$ reported decreased physical activities. About $85 \%$ of subjects reported a negative impact of UI on praying, while $81.6 \%$ reported difficulty travelling for quite long duration i.e.; more than 30 minutes. About $68 \%$ reported affection of ability to do housekeeping. Most of these domains were affected in patients with mixed or urge urinary incontinence, compared with other types of urinary incontinence including praying $(\mathrm{P}=0.024)$, housekeeping $(\mathrm{P}=0.041)$, social activities $(\mathrm{P}=0.009)$, travelling $(\mathrm{P}=0.002)$, and emotional health; in the form of depression/hopelessness $(\mathrm{P}=0.02)$.

Incontinent individuals expressing severe symptoms of UI as detected by high ICIQ-UI score show significant affection of quality of life. Table (4) showing relatively high ICIQ-UI scores were associated with severe impairment of all domains: praying $(\mathrm{P}=0.002)$, housekeeping $(\mathrm{P}<0.001)$, physical recreational activities $\quad(\mathrm{P}<0.001)$, social activities $\quad(\mathrm{P}<0.001)$, travelling (P0.013), feeling of anxiety/frustration $(\mathrm{P}=0.001)$, and feeling of depression/hopelessness $(\mathrm{P}<0.001)$.

Participants with significant LUTS symptoms (as detected by AUA-SI) showed significant affection of 2 QOL domains (praying, emotional health; in the form of depression/hopelessness) as shown in table (5). By qualitative assessment, all domains of QOL were affected in subjects having mild to moderate LUTS, but the quantitative comparative analysis showed a positive correlation between severity of LUTS and severity of QOL affection regarding praying $(\mathrm{P}=0.005)$ and sense of depression $(\mathrm{P}=0.002)$.

A positive correlation was detected between duration of UI and some domains of quality of life. As shown in table (6) physical activities $(\mathrm{P}=0.001)$, social activities $(\mathrm{P}=0.001)$ and travelling $(\mathrm{P}=0.015)$ were the most to be affected.

Table 7 (a and b) represent a bivariate ANOVA analysis for IIQ-7 score as the dependent variable to determine risk factors of decreased UI-associated QOL. A significant positive relationship was found between multiple factors and high scores of IIQ-7: social isolation $(\mathrm{P}<0.001)$, depression $(\mathrm{P}=0.003)$, functional dependence by ADL and IADL $(\mathrm{P}=0.002, \mathrm{P}=0.001)$, advanced frailty status $(\mathrm{P}=0.002)$, severe UI symptoms $(\mathrm{P}<0.001)$, severe LUTS $(\mathrm{P}=0.004)$, long durated UI symptoms $(\mathrm{P}=0.001)$, chronic constipation $(\mathrm{P}=0.021)$ and using alpha-blockers $(\mathrm{P}=0.013)$.

Table (8) shows that social isolation is the only independent predictor for decreased UI-associated QOL, by multivariate linear regression analysis for IIQ-7 score as the dependent variable. 
Table (1): Baseline Characteristics of the Studied Population:

\begin{tabular}{|c|c|c|c|}
\hline \multirow[t]{3}{*}{ Age (years) } & \multirow{3}{*}{$\begin{array}{l}\text { Range } \\
\text { Mean } \pm \text { SD }\end{array}$} & \multicolumn{2}{|c|}{$60-89$} \\
\hline & & \multicolumn{2}{|c|}{$74.550 \pm 7.810$} \\
\hline & & $\mathbf{N}$ & $\%$ \\
\hline \multirow[t]{2}{*}{ Education } & Illiterate & 32 & 53.33 \\
\hline & Educated & 28 & 46.67 \\
\hline \multirow[t]{2}{*}{ Smoking } & Yes & 45 & 75.00 \\
\hline & No & 15 & 25.00 \\
\hline \multirow[t]{2}{*}{ Obesity } & Yes & 20 & 33.33 \\
\hline & No & 40 & 66.67 \\
\hline \multirow[t]{2}{*}{ Marital state } & Married & 48 & 80.00 \\
\hline & Not Married & 12 & 20.00 \\
\hline \multirow[t]{3}{*}{ CFS } & Mild & 23 & $38.33 \%$ \\
\hline & Moderate & 26 & $43.43 \%$ \\
\hline & Severe & 11 & $18.33 \%$ \\
\hline \multirow[t]{2}{*}{ GDS } & Depressed & 34 & $56.67 \%$ \\
\hline & Not depressed & 26 & $43.33 \%$ \\
\hline \multirow[t]{3}{*}{ ADL } & Independent & 19 & $31.66 \%$ \\
\hline & Assisted & 31 & $51.66 \%$ \\
\hline & Dependent & 10 & $16.66 \%$ \\
\hline \multirow[t]{2}{*}{ IADL } & Assisted & 38 & $63.33 \%$ \\
\hline & Dependent & 22 & $36.66 \%$ \\
\hline
\end{tabular}

Obesity; defined as BMI of 30 or more, CFS; Clinical Frailty Scale, GDS; Geriatric depression scale, ADL; Activities of daily livings, IADL: Instrumental activities of daily living.

Table (2): The Prevalence of Different Types of Urinary Incontinence among the Studied Population

\begin{tabular}{lll}
\hline Type of UI & N & $\%$ \\
\hline Urge & 23 & 38.33 \\
Stress & 1 & 1.67 \\
Overflow & 3 & 5.00 \\
Functional & 9 & 15.00 \\
Mixed & 24 & 40.00 \\
Total & 60 & 100.00 \\
\hline
\end{tabular}

UI; Urinary incontinence 
Table (3): The Relationship between Types of Urinary Incontinence and QOL:

\begin{tabular}{|c|c|c|c|c|c|c|c|c|c|c|c|c|c|}
\hline \multirow[t]{3}{*}{ IIQ } & \multirow[t]{3}{*}{ Type of UI } & \multicolumn{10}{|c|}{ IIQ } & \multirow{2}{*}{\multicolumn{2}{|c|}{ Chi-Square }} \\
\hline & & \multicolumn{2}{|c|}{ Never } & \multicolumn{2}{|c|}{ Mild } & \multicolumn{2}{|c|}{ Moderate } & \multicolumn{2}{|c|}{ Severe } & \multicolumn{2}{|c|}{ Total } & & \\
\hline & & $\mathbf{N}$ & $\%$ & $\mathbf{N}$ & $\%$ & $\mathbf{N}$ & $\%$ & $\mathbf{N}$ & $\%$ & $\mathbf{N}$ & $\%$ & $\mathbf{X}^{2}$ & P-value \\
\hline \multirow{5}{*}{ IIQ-1 } & Urge & 7 & 77.78 & 10 & 50.00 & 1 & 6.25 & 5 & 33.33 & 23 & 38.33 & \multirow{5}{*}{23.432} & \multirow{5}{*}{$0.024^{*}$} \\
\hline & Stress & 0 & 0.00 & 0 & 0.00 & 1 & 6.25 & 0 & 0.00 & 1 & 1.67 & & \\
\hline & Overflow & 0 & 0.00 & 0 & 0.00 & 2 & 12.50 & 1 & 6.67 & 3 & 5.00 & & \\
\hline & Functional & 1 & 11.11 & 0 & 0.00 & 5 & 31.25 & 3 & 20.00 & 9 & 15.00 & & \\
\hline & Mixed & 1 & 11.11 & 10 & 50.00 & 7 & 43.75 & 6 & 40.00 & 24 & 40.00 & & \\
\hline \multirow[t]{5}{*}{ IIQ-2 } & Urge & 9 & 47.37 & 12 & 48.00 & 0 & 0.00 & 2 & 28.57 & 23 & 38.33 & \multirow[t]{5}{*}{21.711} & \multirow[t]{5}{*}{$0.041 *$} \\
\hline & Stress & 1 & 5.26 & 0 & 0.00 & 0 & 0.00 & 0 & 0.00 & 1 & 1.67 & & \\
\hline & Overflow & 0 & 0.00 & 3 & 12.00 & 0 & 0.00 & 0 & 0.00 & 3 & 5.00 & & \\
\hline & Functional & 3 & 15.79 & 0 & 0.00 & 4 & 44.44 & 2 & 28.57 & 9 & 15.00 & & \\
\hline & Mixed & 6 & 31.58 & 10 & 40.00 & 5 & 55.56 & 3 & 42.86 & 24 & 40.00 & & \\
\hline \multirow[t]{5}{*}{ IIQ-3 } & Urge & 8 & 66.67 & 11 & 37.93 & 1 & 11.11 & 3 & 30.00 & 23 & 38.33 & \multirow[t]{5}{*}{14.628} & \multirow[t]{5}{*}{0.262} \\
\hline & Stress & 0 & 0.00 & 1 & 3.45 & 0 & 0.00 & 0 & 0.00 & 1 & 1.67 & & \\
\hline & Overflow & 0 & 0.00 & 3 & 10.34 & 0 & 0.00 & 0 & 0.00 & 3 & 5.00 & & \\
\hline & Functional & 2 & 16.67 & 2 & 6.90 & 3 & 33.33 & 2 & 20.00 & 9 & 15.00 & & \\
\hline & Mixed & 2 & 16.67 & 12 & 41.38 & 5 & 55.56 & 5 & 50.00 & 24 & 40.00 & & \\
\hline \multirow[t]{5}{*}{ IIQ-4 } & Urge & 5 & 62.50 & 11 & 45.83 & 0 & 0.00 & 7 & 50.00 & 23 & 38.33 & \multirow[t]{5}{*}{26.566} & \multirow[t]{5}{*}{$0.009 *$} \\
\hline & Stress & 1 & 12.50 & 0 & 0.00 & 0 & 0.00 & 0 & 0.00 & 1 & 1.67 & & \\
\hline & Overflow & 0 & 0.00 & 3 & 12.50 & 0 & 0.00 & 0 & 0.00 & 3 & 5.00 & & \\
\hline & Functional & 1 & 12.50 & 3 & 12.50 & 4 & 28.57 & 1 & 7.14 & 9 & 15.00 & & \\
\hline & Mixed & 1 & 12.50 & 7 & 29.17 & 10 & 71.43 & 6 & 42.86 & 24 & 40.00 & & \\
\hline \multirow{5}{*}{ IIQ-5 } & Urge & 5 & 45.45 & 12 & 60.00 & 1 & 5.88 & 5 & 41.67 & 23 & 38.33 & \multirow{5}{*}{31.236} & $0.002^{*}$ \\
\hline & Stress & 1 & 9.09 & 0 & 0.00 & 0 & 0.00 & 0 & 0.00 & 1 & 1.67 & & \\
\hline & Overflow & 0 & 0.00 & 3 & 15.00 & 0 & 0.00 & 0 & 0.00 & 3 & 5.00 & & \\
\hline & Functional & 3 & 27.27 & 2 & 10.00 & 4 & 23.53 & 0 & 0.00 & 9 & 15.00 & & \\
\hline & Mixed & 2 & 18.18 & 3 & 15.00 & 12 & 70.59 & 7 & 58.33 & 24 & 40.00 & & \\
\hline IIQ-6 & Urge & 5 & 83.33 & 10 & 41.67 & 4 & 22.22 & 4 & 33.33 & 23 & 38.33 & 13.045 & 0.366 \\
\hline & Stress & 0 & 0.00 & 1 & 4.17 & 0 & 0.00 & 0 & 0.00 & 1 & 1.67 & & \\
\hline & Overflow & 0 & 0.00 & 1 & 4.17 & 2 & 11.11 & 0 & 0.00 & 3 & 5.00 & & \\
\hline & Functional & 0 & 0.00 & 2 & 8.33 & 5 & 27.78 & 2 & 16.67 & 9 & 15.00 & & \\
\hline & Mixed & 1 & 16.67 & 10 & 41.67 & 7 & 38.89 & 6 & 50.00 & 24 & 40.00 & & \\
\hline IIQ-7 & Urge & 4 & 100.00 & 9 & 45.00 & 4 & 22.22 & 6 & 33.33 & 23 & 38.33 & 24.096 & $0.020^{*}$ \\
\hline & Stress & 0 & 0.00 & 1 & 5.00 & 0 & 0.00 & 0 & 0.00 & 1 & 1.67 & & \\
\hline & Overflow & 0 & 0.00 & 1 & 5.00 & 2 & 11.11 & 0 & 0.00 & 3 & 5.00 & & \\
\hline & Functional & 0 & 0.00 & 0 & 0.00 & 7 & 38.89 & 2 & 11.11 & 9 & 15.00 & & \\
\hline & Mixed & 0 & 0.00 & 9 & 45.00 & 5 & 27.78 & 10 & 55.56 & 24 & 40.00 & & \\
\hline
\end{tabular}

IIQ-1; Praying, IIQ-2; Housekeeping, IIQ-3; Physical recreational activities, IIQ-4; Social activities, IIQ-5; Travelling. IIQ-6; Anxiety/Frustration, IIQ-7; Depression/Hopelessness 
Table (4): Relationship between Severity of Urinary Incontinence (ICIQ-UI) and QOL:

\begin{tabular}{|c|c|c|c|c|c|c|c|}
\hline & & \multicolumn{4}{|c|}{ ICIQ-Total score } & \multicolumn{2}{|c|}{ ANOVA } \\
\hline & & $\mathbf{N}$ & Mean & \pm & SD & $\mathbf{F}$ & P-value \\
\hline \multirow[t]{4}{*}{ IIQ-1 } & Never & 9 & 7.889 & \pm & 4.457 & \multirow[t]{4}{*}{5.791} & \multirow[t]{4}{*}{$0.002^{*}$} \\
\hline & Mild & 20 & 10.250 & \pm & 4.983 & & \\
\hline & Moderate & 16 & 12.313 & \pm & 4.715 & & \\
\hline & Severe & 15 & 15.467 & \pm & 4.612 & & \\
\hline \multirow[t]{4}{*}{ IIQ-2 } & Never & 19 & 9.579 & \pm & 3.948 & \multirow[t]{4}{*}{7.385} & \multirow[t]{4}{*}{$<0.001 *$} \\
\hline & Mild & 25 & 10.520 & \pm & 5.636 & & \\
\hline & Moderate & 9 & 15.333 & \pm & 3.082 & & \\
\hline & Severe & 7 & 17.429 & \pm & 3.309 & & \\
\hline \multirow[t]{4}{*}{ IIQ-3 } & Never & 12 & 8.167 & \pm & 4.448 & \multirow[t]{4}{*}{11.101} & \multirow[t]{4}{*}{$<0.001 *$} \\
\hline & Mild & 29 & 10.655 & \pm & 4.857 & & \\
\hline & Moderate & 9 & 12.889 & \pm & 3.516 & & \\
\hline & Severe & 10 & 18.200 & \pm & 2.573 & & \\
\hline \multirow[t]{4}{*}{ IIQ-4 } & Never & 8 & 7.750 & \pm & 4.803 & \multirow[t]{4}{*}{9.215} & \multirow[t]{4}{*}{$<0.001 *$} \\
\hline & Mild & 24 & 10.125 & \pm & 4.543 & & \\
\hline & Moderate & 14 & 11.786 & \pm & 4.209 & & \\
\hline & Severe & 14 & 16.786 & \pm & 4.300 & & \\
\hline \multirow[t]{4}{*}{ IIQ-5 } & Never & 11 & 9.727 & \pm & 5.293 & \multirow[t]{4}{*}{3.896} & \multirow[t]{4}{*}{$0.013 *$} \\
\hline & Mild & 20 & 11.150 & \pm & 5.851 & & \\
\hline & Moderate & 17 & 10.765 & \pm & 3.784 & & \\
\hline & Severe & 12 & 16.000 & \pm & 4.348 & & \\
\hline \multirow[t]{4}{*}{ IIQ-6 } & Never & 6 & 7.500 & \pm & 6.221 & \multirow[t]{4}{*}{6.193} & \multirow[t]{4}{*}{$0.001 *$} \\
\hline & Mild & 24 & 9.625 & \pm & 5.037 & & \\
\hline & Moderate & 18 & 14.278 & \pm & 3.997 & & \\
\hline & Severe & 12 & 14.333 & \pm & 4.185 & & \\
\hline \multirow[t]{4}{*}{ IIQ-7 } & Never & 4 & 4.750 & \pm & 1.258 & \multirow[t]{4}{*}{13.292} & \multirow[t]{4}{*}{$<0.001 *$} \\
\hline & Mild & 20 & 8.500 & \pm & 4.407 & & \\
\hline & Moderate & 18 & 13.278 & \pm & 3.893 & & \\
\hline & Severe & 18 & 15.389 & \pm & 4.434 & & \\
\hline
\end{tabular}

ICIQ; International Consultation on Incontinence Questionnaire, UI; Urinary incontinence, IIQ-1; Praying, IIQ-2; Housekeeping, IIQ-3; Physical recreational activities, IIQ-4; Social activities, IIQ-5; Travelling. IIQ-6; Anxiety/Frustration, IIQ-7; Depression/Hopelessness 
Table (5): The Relationship between AUA-SI score and QOL:

\begin{tabular}{|c|c|c|c|c|c|c|c|c|c|}
\hline & \multicolumn{6}{|c|}{ AUA-SI Score } & \multirow{2}{*}{\multicolumn{2}{|c|}{ Chi-Square }} \\
\hline & & \multicolumn{2}{|c|}{ Mild } & \multicolumn{2}{|c|}{ Moderate } & \multicolumn{2}{|c|}{ Total } & & \\
\hline & & $\mathbf{N}$ & $\%$ & $\mathbf{N}$ & $\%$ & $\mathbf{N}$ & $\%$ & $\mathbf{X}^{2}$ & P-value \\
\hline \multirow[t]{4}{*}{ IIQ-1 } & Never & 8 & 15.38 & 1 & 12.50 & 9 & 15.00 & \multirow[t]{4}{*}{12.933} & \multirow[t]{4}{*}{$0.005 *$} \\
\hline & Mild & 19 & 36.54 & 1 & 12.50 & 20 & 33.33 & & \\
\hline & Moderate & 16 & 30.77 & 0 & 0.00 & 16 & 26.67 & & \\
\hline & Severe & 9 & 17.31 & 6 & 75.00 & 15 & 25.00 & & \\
\hline \multirow[t]{4}{*}{ IIQ-2 } & Never & 18 & 34.62 & 1 & 12.50 & 19 & 31.67 & \multirow[t]{4}{*}{6.428} & \multirow[t]{4}{*}{0.093} \\
\hline & Mild & 22 & 42.31 & 3 & 37.50 & 25 & 41.67 & & \\
\hline & Moderate & 8 & 15.38 & 1 & 12.50 & 9 & 15.00 & & \\
\hline & Severe & 4 & 7.69 & 3 & 37.50 & 7 & 11.67 & & \\
\hline \multirow[t]{4}{*}{ IIQ-3 } & Never & 12 & 23.08 & 0 & 0.00 & 12 & 20.00 & \multirow[t]{4}{*}{5.570} & \multirow[t]{4}{*}{0.134} \\
\hline & Mild & 26 & 50.00 & 3 & 37.50 & 29 & 48.33 & & \\
\hline & Moderate & 6 & 11.54 & 3 & 37.50 & 9 & 15.00 & & \\
\hline & Severe & 8 & 15.38 & 2 & 25.00 & 10 & 16.67 & & \\
\hline \multirow[t]{4}{*}{ IIQ-4 } & Never & 8 & 15.38 & 0 & 0.00 & 8 & 13.33 & \multirow[t]{4}{*}{3.338} & \multirow[t]{4}{*}{0.342} \\
\hline & Mild & 22 & 42.31 & 2 & 25.00 & 24 & 40.00 & & \\
\hline & Moderate & 11 & 21.15 & 3 & 37.50 & 14 & 23.33 & & \\
\hline & Severe & 11 & 21.15 & 3 & 37.50 & 14 & 23.33 & & \\
\hline \multirow[t]{4}{*}{ IIQ-5 } & Never & 10 & 19.23 & 1 & 12.50 & 11 & 18.33 & \multirow[t]{4}{*}{2.153} & \multirow[t]{4}{*}{0.541} \\
\hline & Mild & 18 & 34.62 & 2 & 25.00 & 20 & 33.33 & & \\
\hline & Moderate & 13 & 25.00 & 4 & 50.00 & 17 & 28.33 & & \\
\hline & Severe & 11 & 21.15 & 1 & 12.50 & 12 & 20.00 & & \\
\hline \multirow[t]{4}{*}{ IIQ-6 } & Never & 6 & 11.54 & 0 & 0.00 & 6 & 10.00 & \multirow[t]{4}{*}{5.673} & \multirow[t]{4}{*}{0.129} \\
\hline & Mild & 22 & 42.31 & 2 & 25.00 & 24 & 40.00 & & \\
\hline & Moderate & 16 & 30.77 & 2 & 25.00 & 18 & 30.00 & & \\
\hline & Severe & 8 & 15.38 & 4 & 50.00 & 12 & 20.00 & & \\
\hline \multirow[t]{4}{*}{ IIQ-7 } & Never & 4 & 7.69 & 0 & 0.00 & 4 & 6.67 & \multirow[t]{4}{*}{14.808} & \multirow[t]{4}{*}{$0.002^{*}$} \\
\hline & Mild & 20 & 38.46 & 0 & 0.00 & 20 & 33.33 & & \\
\hline & Moderate & 17 & 32.69 & 1 & 12.50 & 18 & 30.00 & & \\
\hline & Severe & 11 & 21.15 & 7 & 87.50 & 18 & 30.00 & & \\
\hline
\end{tabular}

AUA-SI; American Urological Association Symptom Index, IIQ-1; Praying, IIQ-2; Housekeeping, IIQ-3; Physical recreational activities, IIQ-4; Social activities, IIQ-5; Travelling. IIQ-6; Anxiety/Frustration, IIQ-7; Depression/Hopelessness 
Table (6): Relationship between Duration of Urinary Incontinence and QOL:

\begin{tabular}{|c|c|c|c|c|c|c|c|}
\hline \multirow{3}{*}{ IIQ-1 } & \multirow[b]{3}{*}{ Never } & \multicolumn{2}{|c|}{ UI Duration } & \multirow{3}{*}{$\begin{array}{l} \pm \\
\pm\end{array}$} & \multirow{3}{*}{$\begin{array}{l}\text { SD } \\
2.279\end{array}$} & \multicolumn{2}{|c|}{ ANOVA } \\
\hline & & \multirow{2}{*}{$\begin{array}{l}\mathbf{N} \\
9\end{array}$} & \multirow{2}{*}{$\begin{array}{l}\text { Mean } \\
2.722\end{array}$} & & & \multirow{3}{*}{$\begin{array}{l}\mathbf{F} \\
0.365\end{array}$} & \multirow{2}{*}{$\begin{array}{l}\text { P-value } \\
0.778\end{array}$} \\
\hline & & & & & & & \\
\hline & Mild & 20 & 3.050 & \pm & 2.389 & & \multirow{7}{*}{0.087} \\
\hline \multirow{4}{*}{ IIQ-2 } & Moderate & 16 & 3.625 & \pm & 2.187 & \multirow{6}{*}{2.305} & \\
\hline & Severe & 15 & 3.100 & \pm & 2.037 & & \\
\hline & Never & 19 & 3.026 & \pm & 2.300 & & \\
\hline & Mild & 25 & 2.540 & \pm & 2.096 & & \\
\hline \multirow{5}{*}{ IIQ-3 } & Moderate & 9 & 4.222 & \pm & 2.048 & & \\
\hline & Severe & 7 & 4.429 & \pm & 1.813 & & \\
\hline & Never & 12 & 1.875 & \pm & 1.539 & \multirow[t]{4}{*}{5.854} & \multirow[t]{4}{*}{$0.001 *$} \\
\hline & Mild & 29 & 2.845 & \pm & 2.228 & & \\
\hline & Moderate & 9 & 5.333 & \pm & 1.732 & & \\
\hline \multirow{3}{*}{ IIQ-4 } & Severe & 10 & 3.700 & \pm & 1.767 & & \\
\hline & Never & 8 & 2.125 & \pm & 1.808 & \multirow[t]{4}{*}{6.653} & \multirow[t]{4}{*}{$0.001 *$} \\
\hline & Mild & 24 & 2.125 & \pm & 2.060 & & \\
\hline \multirow{6}{*}{ IIQ-5 } & Moderate & 14 & 4.500 & \pm & 1.605 & & \\
\hline & Severe & 14 & 4.214 & \pm & 2.082 & & \\
\hline & Never & 11 & 2.636 & \pm & 2.111 & \multirow[t]{4}{*}{3.780} & \multirow[t]{4}{*}{$0.015^{*}$} \\
\hline & Mild & 20 & 2.150 & \pm & 2.289 & & \\
\hline & Moderate & 17 & 3.941 & \pm & 1.713 & & \\
\hline & Severe & 12 & 4.250 & \pm & 2.050 & & \\
\hline \multirow[t]{4}{*}{ IIQ-6 } & Never & 6 & 2.583 & \pm & 2.333 & \multirow[t]{4}{*}{2.663} & \multirow[t]{4}{*}{0.057} \\
\hline & Mild & 24 & 2.542 & \pm & 2.141 & & \\
\hline & Moderate & 18 & 3.250 & & 2.225 & & \\
\hline & Severe & 12 & 4.583 & \pm & 1.730 & & \\
\hline \multirow[t]{4}{*}{ IIQ-7 } & Never & 4 & 2.125 & \pm & 2.016 & \multirow[t]{4}{*}{1.789} & \multirow[t]{4}{*}{0.160} \\
\hline & Mild & 20 & 2.450 & \pm & 2.276 & & \\
\hline & Moderate & 18 & 3.528 & \pm & 2.317 & & \\
\hline & Severe & 18 & 3.833 & & 1.855 & & \\
\hline
\end{tabular}

IIQ-1; Praying, IIQ-2; Housekeeping, IIQ-3; Physical recreational activities, IIQ-4; Social activities, IIQ-5; Travelling. IIQ-6; Anxiety/Frustration, IIQ-7; Depression/Hopelessness 
Table (7a): Bivariate ANOVA analysis for IIQ-7 score:

\begin{tabular}{|c|c|c|c|c|c|c|c|}
\hline & & IIQ & Total sc & & & T-Test & ANOVA \\
\hline & & $\mathbf{N}$ & Mean & \pm & SD & T or $\mathbf{F}$ & P-value \\
\hline Obesity & No & 40 & 9.850 & \pm & 5.294 & -1.155 & 0.253 \\
\hline & Yes & 20 & 11.450 & \pm & 4.536 & & \\
\hline Social isolation & Yes & 14 & 15.714 & \pm & 3.539 & 5.488 & $<0.001^{*}$ \\
\hline & No & 46 & 8.761 & \pm & 4.311 & & \\
\hline GDS & Depressed & 34 & 12.059 & \pm & 4.729 & 3.137 & $0.003^{*}$ \\
\hline & Not depressed & 26 & 8.192 & \pm & 4.733 & & \\
\hline ADL & Independent & 19 & 7.579 & \pm & 4.788 & 6.720 & $0.002 *$ \\
\hline & Assisted & 31 & 10.935 & \pm & 4.524 & & \\
\hline & Dependent & 10 & 14.000 & \pm & 4.738 & & \\
\hline IADL & Assisted & 38 & 8.763 & \pm & 4.907 & -3.558 & $0.001 *$ \\
\hline & Dependent & 22 & 13.182 & \pm & 4.113 & & \\
\hline CFS & Mild & 23 & 7.609 & \pm & 5.097 & 6.874 & $0.002 *$ \\
\hline & Moderate & 26 & 11.808 & \pm & 3.826 & & \\
\hline & Severe & 11 & 12.818 & \pm & 5.344 & & \\
\hline ICIQ-UI & Mild & 9 & 5.111 & \pm & 3.887 & 16.137 & $<0.001 *$ \\
\hline & Moderate & 22 & 8.409 & \pm & 3.347 & & \\
\hline & Severe & 23 & 12.652 & \pm & 4.344 & & \\
\hline & Very severe & 6 & 16.833 & \pm & 2.927 & & \\
\hline AUA Score & Mild & 52 & 9.654 & \pm & 5.009 & -3.031 & $0.004^{*}$ \\
\hline & Moderate & 8 & 15.125 & \pm & 2.100 & & \\
\hline Type of UI & Urge & 23 & 8.261 & \pm & 5.553 & 2.469 & 0.055 \\
\hline & Stress & 1 & 5.000 & \pm & 0.000 & & \\
\hline & Overflow & 3 & 9.667 & \pm & 0.577 & & \\
\hline & Functional & 9 & 12.333 & \pm & 5.362 & & \\
\hline & Mixed & 24 & 12.000 & \pm & 4.054 & & \\
\hline HTN & Yes & 32 & 11.000 & \pm & 5.042 & 1.007 & 0.318 \\
\hline & No & 28 & 9.679 & \pm & 5.107 & & \\
\hline Chronic liver disease & Yes & 7 & 7.857 & \pm & 6.067 & -1.414 & 0.163 \\
\hline & No & 53 & 10.717 & \pm & 4.897 & & \\
\hline Congestive heart failure & Yes & 15 & 10.600 & \pm & 5.262 & 0.189 & 0.850 \\
\hline & No & 45 & 10.311 & \pm & 5.067 & & \\
\hline DM & Yes & 29 & 11.103 & \pm & 4.065 & 1.065 & 0.291 \\
\hline & No & 31 & 9.710 & \pm & 5.849 & & \\
\hline Thyroid & Yes & 4 & 13.500 & \pm & 5.260 & 1.279 & 0.206 \\
\hline & No & 56 & 10.161 & \pm & 5.034 & & \\
\hline OA & Yes & 26 & 9.885 & \pm & 4.141 & -0.663 & 0.510 \\
\hline & No & 34 & 10.765 & \pm & 5.716 & & \\
\hline Lumbar spondylosis & Yes & 7 & 13.571 & \pm & 3.690 & 1.803 & 0.077 \\
\hline & No & 53 & 9.962 & \pm & 5.106 & & \\
\hline Stroke & Yes & 11 & 11.364 & \pm & 4.523 & 0.706 & 0.483 \\
\hline & No & 49 & 10.163 & \pm & 5.206 & & \\
\hline Recurrent UTI & Yes & 8 & 11.875 & \pm & 3.796 & 0.892 & 0.376 \\
\hline & No & 52 & 10.154 & \pm & 5.233 & & \\
\hline Renal stones & Yes & 2 & 10.000 & \pm & 1.414 & -0.108 & 0.915 \\
\hline & No & 58 & 10.397 & \pm & 5.157 & & \\
\hline CKD & Yes & 14 & 12.500 & \pm & 5.125 & 1.818 & 0.074 \\
\hline & No & 46 & 9.739 & \pm & 4.933 & & \\
\hline $\mathrm{BPH}$ & Yes & 34 & 11.147 & \pm & 4.698 & 1.343 & 0.185 \\
\hline & No & 26 & 9.385 & \pm & 5.456 & & \\
\hline Chronic constipation & Yes & 29 & 11.931 & \pm & 4.415 & 2.374 & $0.021 *$ \\
\hline & No & 31 & 8.935 & \pm & 5.285 & & \\
\hline ACEI & Yes & 17 & 12.118 & \pm & 5.476 & 1.691 & 0.096 \\
\hline & No & 43 & 9.698 & \pm & 4.798 & & \\
\hline Diuretic & Yes & 18 & 11.944 & \pm & 5.263 & 1.580 & 0.119 \\
\hline & No & 42 & 9.714 & \pm & 4.900 & & \\
\hline Antidepressants & Yes & 7 & 11.571 & \pm & 6.294 & 0.656 & 0.514 \\
\hline & No & 53 & 10.226 & \pm & 4.941 & & \\
\hline Alpha blockers & Yes & 17 & 12.941 & \pm & 4.479 & 2.570 & $0.013^{*}$ \\
\hline & No & 43 & 9.372 & \pm & 4.981 & & \\
\hline Alpha reductase inhibitor & Yes & 10 & 11.000 & \pm & 4.761 & 0.418 & 0.677 \\
\hline & No & 50 & 10.260 & \pm & 5.170 & & \\
\hline Anticholinergics & Yes & 2 & 11.500 & \pm & 9.192 & 0.314 & 0.754 \\
\hline & No & 58 & 10.345 & \pm & 5.011 & & \\
\hline
\end{tabular}


Table (7 b): Bivariate correlation analysis for IIQ-7 score:

\begin{tabular}{ccc}
\hline & \multicolumn{2}{c}{ IIQ-7 Total score } \\
\cline { 2 - 3 } & $\mathbf{r}$ & P-value \\
UI Duration & 0.404 & $0.001^{*}$ \\
ICIQ-Total & 0.703 & $<0.001^{*}$ \\
Number of drugs & 0.229 & 0.095 \\
Charlson comorbidity index & 0.049 & 0.712 \\
\hline
\end{tabular}

Table (8): Multivariate linear regression analysis for predictors of QOL (IIQ-7 score) in older frail males with UI :

\begin{tabular}{cccccc}
\hline & \multicolumn{2}{c}{$\begin{array}{c}\text { Unstandardized } \\
\text { Coefficients }\end{array}$} & $\begin{array}{c}\text { Standardized } \\
\text { Coefficients }\end{array}$ & T & P-value \\
\cline { 2 - 4 } & B & Std. Error & Beta & & \\
Social isolation & -4.585 & 1.399 & -0.386 & -3.277 & $0.002^{*}$ \\
GDS & -0.724 & 1.186 & -0.071 & -0.610 & 0.545 \\
ADL & -1.018 & 1.299 & -0.137 & -0.784 & 0.437 \\
IADL & 1.171 & 1.446 & 0.112 & 0.810 & 0.422 \\
\hline CFS & 0.529 & 1.168 & 0.076 & 0.453 & 0.652 \\
\hline ICIQ-UI & 0.845 & 1.624 & 0.145 & 0.520 & 0.605 \\
\hline AUA Score & 2.110 & 1.631 & 0.143 & 1.294 & 0.202 \\
Chronic constipation & -2.073 & 1.083 & -0.206 & -1.914 & 0.062 \\
\hline Alphablockers & 0.269 & 1.319 & 0.024 & 0.204 & 0.839 \\
UI Duration & 0.144 & 0.256 & 0.062 & 0.563 & 0.576 \\
\hline ICIQ-Total & 0.193 & 0.287 & 0.201 & 0.670 & 0.506 \\
\hline
\end{tabular}

\section{Discussion}

The influence of UI on different QOL domains is wellknown. The current study tries to pinpoint this relation and to determine the controlling factors in frail elderly males who have been underestimated in literature concerning UI. UI is considered a silent disease in elderly men, and this may negatively affect physical and psychosocial health in this population (Griebling et al., 2008) [10]. Besides, the co-existence of frailty and UI syndromes in older individuals have deleterious effects on health-related QOL (Carlos, 2012) [27].

In the current study, we found subjects with severe incontinence symptoms (especially mixed forms or pure urge UI), long UI symptoms duration and relatively severe LUTS, showing marked affection of healthrelated quality of life. Psychological and religious life domains were the most to be affected.

The most prevalent type of UI among the studied population was mixed forms of UI $(40 \%$ of participants) with approximate prevalence of urge UI $(38.3 \%)$.

It is worthwhile mentioning that, in our study, mixed UI was statistically analyzed as the combination of 2 or more types of UI. In most participants, it was actually the combination of urge and functional UI. The large percentage of mixed UI was expected in this frail cohort with high mean age, impaired functional level and associated predisposing factors as associated comorbidities and medications.

Most of the studies have shown that urge UI is the prevalent type of UI among elderly males (Griebling, 2008) [10]. In a prospective cohort study performed by Wehrberger et al. (2011) [28] to assess LUTS and UI in geriatric population, it was found that $50 \%$ of men had overactive bladder (OAB) symptoms (29\% OABdry and $21 \%$ OAB-wet). Another study conducted by Aniuliene et al. (2016) [29], regarding types of UI among primary care patients, showed that $60 \%$ of men were classified as having pure urge UI. Teunissen et al. (2009) [30] study showed that $70 \%$ of men recruited had urge UI and $11 \%$ had stress UI. A mailed survey done by Song et al. (2007) [31] revealed that urge UI was the most prevalent subtype (44.6\%) among men reporting this condition. In the study of Wang et al. (2017) [32], 440 men aging 80 years and older were 
recruited, where the overall prevalence of UI was $19.1 \%$ (mostly urge UI: $51.2 \%$ and functional UI: $41.7 \%)$.

In a systemic review (which included a total of 20 studies) conducted by Shamliyan et al. (2009) [33], it was found that urge UI was the most prevalent type of UI; $11.7 \%$ in those older than 65 years. Urge UI is one of the main storage symptoms caused by detrusor overactivity; which is the most common form of bladder dysfunction encountered in older males. (Lee et al., 2017) [34].

Previous studies had demonstrated the impact of UI in affecting QOL in older adults by using general tools in continent versus incontinent subjects, with enrollment of cofounding factors as baseline function, cognition and comorbid conditions (DuBeau et al., 2006) [7] (Ko et al., 2005) [35]. Using a disease-specific healthrelated QOL questionnaire in the current study was more sensitive in measuring this relationship in frail elderly. In our study, about $80-90 \%$ of incontinent subjects experienced impaired QOL with varying degrees. Most of participants reported affection of emotional wellbeing (feelings of depression, hopelessness and frustration) and more than two thirds had problems with travelling, outside physical activities and social participation.

This agrees with a cross-sectional survey performed by Teunissen et al. (2006) [30] to study the impact of UI on daily life in community dwelling elderly using IIQ that showed that $37 \%$ of patients reported restricted transportation, $10 \%$ were restricted in their physical activities and up to half of them felt nervous, embarrassed and frustrated. The difference in proportions of subjects affected in each domain can be explained by the discrepancy in baseline characters of study populations. In the study of Teunissen et al. (2006) [30], the impact score for some items were significantly higher in men than in women, and men compromised only $15.8 \%$ of study group. Furthermore, urge UI is more bothersome than stress UI, which is the predominant type in our study population.

Nearly $85 \%$ of participants reported a negative impact of UI on praying, which is actually not an item in the original IIQ and was added in the Arabic version to suit our population. Praying is a unique feature of Muslims daily life activities. Other studies had explored the impact of UI on praying. In the study of El-Azab et $\boldsymbol{a l}$. (2007) [36] 90\% of incontinent group were distressed by their restricted ability to pray. Another study conducted by Van den Muijsenberg and Lagro-Jansse (2006) [37] to examine the impact of urinary incontinence on daily lives of Moroccan and Turkish women, showed that most of participants had difficulties with praying and maintaining ritual purification. Both of these studies recruited adult women, whereas data concerning older men population was deficient.

The previously mentioned domains were affected in patients with mixed or urge urinary incontinence, compared with other types of urinary incontinence. That was in agreement the findings of Coyne et al. (2003) [38], who examined the impact of each type of UI (stress, urge, and mixed) on health-related QOL in both sexes using OAB-q (a health-related QOL scale for over $\mathrm{OAB}$ ). It was found that mixed UI and urge UI groups reported significantly lower health-related QOL $(P<0.02)$ and $(\mathrm{P}<0.001)$ respectively and greater symptom bother $(P<0.001)$ in both groups than the stress UI group. The previous findings were explained by the unpredictable nature of urge incontinence and less efficient coping mechanisms (Coyne et al., 2003) [38]. In the same way, a cross-sectional study, applied in residential homes on a total of 1110 elderly by Aslan et al. (2009) [35], showed that urge UI had more impact on the QOL than that of stress or functional UI.

A population-based cohort study by Wehrberger et al. (2012) [28], assessing LUTS and UI in elderly subjects, revealed that $46 \%$ of the male cohort stated that their QOL was affected by LUTS and UI, such that nocturia was the most bothersome symptom $(29.7 \%)$, followed by daytime frequency $(9.5 \%)$, strong uncontrollable urgency $(4.1 \%)$ and UI $(2.7 \%)$. Furthermore, the study showed that individuals with pure urge UI or mixed UI reported a greater negative impact on QOL than those with pure stress UI.

Participants with severe symptoms of UI (regarding amount, frequency and subjective impact as detected by ICIQ-UI) showed severe affection of QOL in all domains compared to those with mild to moderate symptoms. In the study of a Aguilar-Navarro et al. (2012) [40] assessing QOL by the Short Form Healthy Survey questionnaire (SF-36) in an elderly group, participants with severe UI symptoms (by the Sandvik Severity Index) showed lower scores on the physical component summary of the SF-36.

UI is one of the diverse LUTS experienced by the elderly populations. The LUTS can be voiding symptoms (intermittency, weak stream, straining) and/or storage (frequency, urgency, urge UI and nocturia) and post-micturitional (incomplete emptying and post-void dribbling). In the current study, incontinent subjects were further assessed by AUA-SI. Patients with significant LUTS (as detected by AUASI) showed significant affection of emotional health; in the form of depression/hopelessness $(\mathrm{P}=0.02)$ besides affection of praying domain $(\mathrm{P}=0.05)$ as detected by IIQ-7.

In the EPIC study (Irwin et al., 2009) [41] men with $\mathrm{OAB}$ symptoms were more likely to experience multiple LUTS subtypes including urgency, nocturia, terminal dribble and sensation of incomplete emptying. Authors reported that LUTS severity may have been underestimated by the AUA-SI as it does not assess UI in details. The most reported symptom was urgency followed by nocturia. A subset of men expressed symptom bother due to $\mathrm{OAB}$, that increased with urgency severity and severity and number of associated LUTS, which comes in agreement with our findings. 
Emotional health impact was a significantly prominent feature in patients experiencing LUTS and UI, where a positive relationship can be outlined through assessment with different tools. More than two thirds of our study group reported emotional affection which is a subjective sense of depression and hopelessness. Subjects were screened by GDS-15 to obtain an objective assessment for depression, and $56.67 \%$ were found to be depressed. This agrees with the study of Enberg et al. (2001) [42] performed on a group of homebound males and females elders with UI, where the prevalence of depression found to be $50 \%$ by GDS15.

Our findings also agreed with the cross-sectional study done by Wang et al. (2017) [43], where subjects with UI expressed more depressive symptoms (as assessed by GDS-5, $\mathrm{p}=0.02$ ). In a study performed by Tamanini et al. (2009) [44] on a Brazilian elderly population for the analysis of factors influencing UI, $34 \%$ of incontinent individuals reported depression and it was observed that having moderate or severe depression increased the chance of UI presentation by a factor of 2.49 for both sexes. This agrees with the hypothesis that these two conditions might have the same biological basis. In the study of Chen et al. (2009) [45], a population of elderly institutionalized Chinese males were evaluated by the GDS-15 and the Minimum Data Set (MDS) tool, and revealed that incontinent subjects had statistically significant depressive symptoms.

Participants experiencing UI symptoms for relatively long durations showed affection of physical recreational activities, social activities and travelling domains. This agrees with the findings of Iglesias et al. (2000) [46], where durations of more than 5 years of UI symptoms were associated with negative impact on incontinent subjects' lifestyles $(\mathrm{OR}=2.30)$.

UI-associated QOL was found to be affected by some cofounding factors in the current study including social and psychological factors, functional level, frailty status, comorbidity (chronic constipation), medication use (i.e. alpha-blockers), the presence of other LUTS and severity and duration of UI symptoms. Multivariate linear regression analysis revealed that social isolation is an independent predictor of impaired UI-associated QOL. These findings reflect the complex underlying precipitating factors of UI which may affect QOL in older frail males through direct and indirect pathways.

Previous studies have demonstrated the relationship between UI and social isolation, as UI has been found to be associated with loneliness. This may result from social avoidance secondary to feelings of shame, embarrassment, and decreased self-confidence (Stickly et al., 2017) [47]. On the other hand, social isolation was found to exert negative impact on the health and behavioral habits as well as the psychological and cognitive wellbeing (Nicholson, 2012), thus affecting QOL in older adults. In the study of Fultz and Herzog (2001) [48], aimed at understanding this relationship, subjects with higher scores of social desirability reported less impact of UI. This agrees with our findings where social isolation was positively associated with worse UI-associated QOL.

The influence of functional impairment on QOL in patients with UI had been demonstrated by other studies. In the study of Iglesias et al. (2000) [46], subjects with mild disability showed a greater impact of UI on their lifestyles according to multivariate analysis $(\mathrm{OR}=2.6)$ and in the study of DuBeau et al. (2006) [7], poorer UI-associated QOL was noticed in residents with moderate ADL impairment.

More advanced frailty status was positively related to worse UI-associated QOL. Frailty had been related to lower physical capacity and impaired QOL dimensions (Langlois et al., 2012) [50]. This may precipitate functional UI and affect overall QOL in incontinent patients. In continuum with the findings of Chong $\boldsymbol{e t}$ al. (2018) [51], where frailty was found to be a predictor for UI, frailer status may be a predictor for more severe UI thus more impaired QOL.

In the study of Aslan et al. (2009) [39], the influence on UI-associated QOL was explored. The dependent variable was the QOL assessed by King's health questionnaire. Covariates were age, gender, cognitive function, comorbid conditions, and associated LUTS as urgency and frequency. It was found that the presence of comorbid conditions was associated with worse QOL in UI $(\mathrm{P}=0.002)$. Our findings were distinct regarding the effect of chronic diseases on QOL in UI. This may be explained by the subjective perspectives of QOL in frail elderly, where frail individuals reported social contacts as the most important factor for quality of life, while non-frail reported health as the most important (Puts et al.,2007) [6].

The effect of comorbidities on UI in frail elderly is well identified in literature (Fonda et al., 2005) [2]. In chronic constipation, rectal distention may exacerbate LUTS, including UI (Tannenbaum et al., 2013) [52]. Furthermore, previous studies have found that chronic constipation exerts negative effect on QOL in older adults (Norton, 2013) [53].

The positive relationship between alpha-blockers use and impaired UI-associated QOL may be related to the increased severity of UI symptoms and frequent use of alpha-blockers. Alpha-blockers are one of the main treatment lines used in UI secondary to bladder outlet obstruction (Jarvis et al., 2014) [54].

According to our findings, UI exerts a negative influence on QOL through several ways including duration and severity of UI symptoms, UI resultant adverse effects (depression and social isolation) and UI risk factors (functional level, frailty status, comorbid conditions). These factors may interact together potentiating the negative impact on QOL.

Although total cure of UI in frail older people is challenging due to its multifactorial complex etiology, but addressing the fore-mentioned factors through nonpharmacological and pharmacological measures may 
improve different aspects of quality of life. QOL is considered as a "central concern" for frail people (Fonda et al., 2005) [2]. This can be supported by the findings of our study, while other prospective studies may be needed to demonstrate and measure the effect of treatment of UI on QOL in frail older male patients. The strength of this study is that it uncovered data concerning the impact of UI on QOL in an understudied population i.e. frail elderly males and it used quantitative methods for analyzing the problem. New aspects were explored in this frail population including the severity and duration of UI symptoms and their relation to the degree of QOL affection as well as the effect of cofounding factors on UI-associated QOL. These factors can be targets for therapy to improve QOL in these patients. The limitations of this study is the use of a disease-related questionnaire which didn't allow the assessment of QOL in continent counterparts.

\section{Conclusion:}

UI exerts negative impact on all domains of QOL of frail older males with varying degrees. Improving risk factors of UI, severity of symptoms, social and psychological effects of UI are important targets to enhance QOL in this group.

Ethical considerations

Informed consent was taken from every older male participating in this study. The study methodology was reviewed and approved by the Research Review Board of the Geriatrics and Gerontology Department, Faculty of Medicine, Ain Shams University.

Disclosure Statement

There is no conflict of interest.

References:

1. Prud'homme G, Alexande L, Orme S (2018): Management of urinary incontinence in frail elderly women. Obstetrics, Gynaecology and Reproductive Medicine; 28(2): 39-45.

2. Fonda D, DuBeau CE, Harari D, Ouslander JG, Palmer M, Roe B (2005). APA citation [Online]. Incontinence in the Frail Elderly. Available https://www.ics.org/Publications/ICl_3/v2.pdf/chap18.pdf [Accessed 7 Mar. 2020].

3. Kojima G, Liljas AE, lliffle $S$ (2019). Frailty syndrome: implications and challenges for health care policy. Risk Management and Healthcare Polic; 12: 23-30.

4. Wagg A, Gibson W, Ostaszkiewicz J, Johnson T, Markland A Palmer M, et al. (2014). Urinary incontinence in frail elderly persons: Report from the 5th International Consultation on Incontinence. Neurourology and Urodynamics, 34(5), pp.398406 .

5. Kwong E, Lai C and Liu F (2014). Quality of life in nursing home settings: Perspectives from elderly residents with frailty. Clinical Nursing Studies, 2(1).

6. Puts $M$, Shekary N, Widdershoven G, Heldens J, Lips $P$ and Deeg $D$ (2006). What does quality of life mean to older frail and non-frail community-dwelling adults in the Netherlands?. Quality of Life Research, 16(2), pp.263-277.

7. DuBeau C, Simon S and Morris J (2006). The Effect of Urinary Incontinence on Quality of Life in Older Nursing Home Residents. Journal of the American Geriatrics Society, 54(9), pp.1325-1333.

8. Dugger B (2010). Concept Analysis of Health-Related Quality of Life in Nursing Home Residents with Urinary Incontinence. Urologic Nursing, 30(2), p.112.

9. Anger JY, Saigal CS, Stothers L, Thom DV, Rodriguez LV, Litwin MS (2006). The prevalence of urinary incontinence among community dwelling men: Results from the National Health and
Nutrition Examination Survey. THE JOURNAL OF UROLOGY; vol. 176, 2103-2108, DOI:10.1016/j.juro.2006.07.029.

10. Griebling TL (2008). Urinary incontinence and voiding dysfunction in elderly men. Current Bladder Dysfunction Reports, 3(4), pp. 241-246.

11. Leduc JM, Mehta KM \& Covinsky KE (2004): Urinary incontinence and its association with death, nursing home admission and functional decline. J Am Geriatr Soc; 52:712718.

12. Folstein MF, Folstein SE, McHugh PR (1975): "Mini-mental state". A practical method for grading the cognitive state of patients for the clinician. J Psychiatr Res.; 12(3):189-98.

13. El-OkI MA, Elbanoby MH, Eletrby MA, Mortagy AK and Elsaied MN (2002): Prevalence of Alzheimer dementia and other causes of dementia in Egyptian elderly. MD thesis, Faculty of Medicine, Ain Shams University. Geriatric Department Library.

14. Rockwood K, Song X, MacKnight C, Bergman H, Hogan DB, McDowell I, et al. (2005): A global clinical measure of fitness and frailty in elderly people. CMAJ; 173(5):489-95.

15. Juma S, Taabazuing MM, Montero-Odasso M (2016): Clinical Frailty Scale in an Acute Medicine Unit: a Simple Tool That Predicts Length of Stay. Can Geriatr J; 19(2):34-9.

16. Katz $S$, Ford $A B$, Moskowitz RW, Jackson BA and Jaffe MW (1963): Studies of illness in the aged. The index of ADL: $A$ standardized measure of biological and psychosocial function. JAMA; 21(9); 185:914-9.

17. Lawton MP, Brody EM (1969): Assessment of older people: selfmaintaining and instrumental activities of daily living. Gerontologist; 9(3): 179-186.

18. Shehata AS, Elbanoby MH, Mortagy AK and Ghanem M (1998): Prevalence of depression among Egyptian Geriatric Community. Ain Shams University. Geriatric Department Library.

19. Sheikh JA and Yesavage YA (1986): Geriatric Depression Scale. Recent evidence and development of a shorter version. Clinica Gerontology: A Guide to Assessment and Intervention; 165-173, NY: The Haworth Press.

20. Lagro-Janssen ALM, Breedveldt Boer HP van Dongen JJ, Lemain T, et al. NHG-standaard: incontinentie voor urine Dutch College Guidelines on Urinary Incontinence] (1995). Huisarts Wet; 38:71-80

21. D'Ancona CD, Haylen BT, Oelke M, Herschorn S, AbranchesMonteiro L, Arnold EP, Goldman HB, Hamid R, Homma $Y$, Marcelissen T, Rademakers K, Schizas A, Singla A, Soto I, Tse $V$, de Wachter S. An International Continence Society (ICS) Report on the Terminology for Adult Male Lower Urinary Tract and Pelvic Floor Symptoms and Dysfunction. Neurourol Urodyn. 2019 DOI: 10.1002/nau.23897

22. Hashim H, Avery K, Mourad MS, et al. (2006): The Arabic ICIQ-UI SF: An Alternative Language Version of the English ICIQ-UI SF, Neurourology and Urodynamics 25:277-282Teunissen D, Bosch WV, Weel CV, Janssn TL (2006). "It can always happen": The impact of urinary incontinence on elderly men and women. Scandinavian Journal of Primary Health Care, 24:3, 166-173, DOI: $10.1080 / 02813430600739371$

23. Barry M, Fowler F, O'Leary M, Bruskewitz $R$, Holtgrewe $H$, Mebust $W$ and Cockett A. (1992). The American Urological Association Symptom Index for Benign Prostatic Hyperplasia. Journal of Urology, 148(5 Part 1), pp.1549-1557.

24. El-Azab and Mascha (2009): Arabic Validation of the Urogenital Distress Inventory and Adapted Incontinence Impact Questionnaires-Short Forms. Neurourology and Urodynamics; 28:33-39.

25. Charlson ME, Pompei $P$, Ales KL, et al. A new method of classifying prognostic comorbidity in longitudinal studies: development and validation. J Chronic Dis. 1987;40(5): 373-383.

26. Quan H, Li B, Couris CM, Fushimi K, Graham Pm Hider P, Januel $J M$, Sundararajan $V$ (2011). Updating and validating the Charlson comorbidity index and score for risk adjustment in hospital discharge abstracts using data from 6 countries. Am J Epidemiol;173:676-682

27. Carlos VB (2012)." Geriatric urinary incontinence - special concerns on the frail elderly" in Alhasso A \& Fernando A (ed.) Urinary incontinence. Rijeka, Croatia: In Tech, pp. 113-130

28. Wehrberger C, Madersbacher S, Jungwirth S, Fischer P, Tragel $K H$ (2012). Lower urinary tract symptoms and urinary incontinence in a geriatric cohort - a population-based analysis. BJU INTERNATIONAL: 110; 1516-1521, doi:10.1111/j.1464-410X.2012.11022.x

29. Aniuliene R, Aniulis $P$, Steibliene V (2016). Risk Factors and types of urinary incontinence among middle-aged and older 
male and female primary care patients in Kaunas region of Lithuania: Cross Sectional Study. Miscellaneous: Vol 13 No 01; 2553.

30. Teunissen D, Bosch WV, Weel CV, Janssn TL (2006). "It can always happen": The impact of urinary incontinence on elderly men and women. Scandinavian Journal of Primary Health Care, 24:3, 166-173, DOI: 10.1080/02813430600739371.

31. Song HJ, Bae JM (2007). Prevalence of urinary incontinence and lower urinary tract symptoms for community-dwelling elderly 85 years of age and older. J Wound Ostomy Continence Nurs , 34:535-541.

32. Wang CJ, Hung CH, Tang TC, Chen LY, Peng LN, Hsiao FY, et al. (2017). Urinary Incontinence and Its Association with Frailty Among Men Aged 80 Years or Older in Taiwan: A CrossSectional Study. Rejuvenation Res; 20(2):111-117.

33. Shamliyan TA, Wyman JF, Ping R, Wilt TJ, Kane RL (2009). Male urinary incontinence: prevalence, risk factor and preventive interventions. REVIEWS IN UROLOGY: VOL. 11; NO. 3.

34. Lee CL, Kuo HC (2017). Pathophysiology of benign prostate enlargement and lower urinary tract symptoms: Current concepts. Tzu Chi Medical Journal; 29(2): 79-83.

35. Ko Y, Lin SJ, Salmon JW, Bron MS (2005). The impact of urinary incontinence on quality of life of the elderly. Am J Manag Care;11:S103-S111.

36. El-Azab, Mohamed EM, \& Sabra HI (2007). The prevalence and risk factors of urinary incontinence and its influence on the quality of life among Egyptian women. Neurourol. Urodynam; 26:783-788.

37. Van den Muijsenberg ME, Lagro-Janssen TA (2006): Urinary incontinence in Moroccan and Turkish women: a qualitative study on impact and preferences for treatment. Br J Gen Pract; 56(533):945-9.

38. Coyne KS, Zhou Z, Thompson C, Versi E (2003). The impact on health-related quality of life of stress, urge and mixed urinary incontinence. BJU INTERNATIONAL; 9 2: $73 \quad-735$, doi:10.1046/j.1464-410X.2003.04463.x

39. Aslan E, Beji NK, Erkan HA, Yalcin O, Gungor F (2009). Urinary incontinence (UI) and quality of life $(Q \circ L)$ of the elderly residing in residential homes in Turkey. Archives of Gerontology and Geriatrics; Vol 49, Issue 2, p 304-310.

40. Aguilar-Navarro S, Navarrete-Reyes A, Grados-Chavarria B, Garcia-Lara J, Amieva $H$ and Avila-Funes J (2012). The Severity of Urinary Incontinence Decreases Health-Related Quality of Life among Community-Dwelling Elderly. The Journals of Gerontology Series A: Biological Sciences and Medical Sciences, 67(11), pp.1266-1271.

41. Irwin DE, Milsom I, Kopp Z, Aribani W, HERschorn S (2009). Prevalence, severity, and symptom bother of lower urinary tract symptoms among men in the EPIC Study: Impact of overactive bladder. European Urology; Vol 56, Issue 1: 14-20.

42. Engberg $S$, Sereika $S$, Weber E, Engberg $R, M c$ Dowell $B$ and Reynolds C (2001). Prevalence and Recognition of Depressive Symptoms Among Homebound Older Adults with Urinary Incontinence. Journal of Geriatric Psychiatry and Neurology, 14(3), pp.130-139.

43. Wang CJ, Hung CH, Tang TC, Chen LY, Peng LN, Hsiao FY, et al. (2017). Urinary Incontinence and Its Association with Frailty Among Men Aged 80 Years or Older in Taiwan: A Cross. Sectional Study. Rejuvenation Res; 20(2):111-117.

44. Tamanini J T, Lebrão $M L$, Duarte $Y A$, Santos JL, \& Laurenti $R$ (2009). Analysis of the prevalence of and factors associated with urinary incontinence among elderly people in the Municipality of São Paulo, Brazil: SABE Study (Health, Wellbeing and Aging). Cadernos De Saúde Pública, 25(8), 17561762. doi: 10.1590/s0102-311x2009000800011.

45. Chen YM, Hwang SJ, Chen LK, Chen DY, \& Lan CF (2009). Urinary incontinence among institutionalized oldest old Chinese men in Taiwan. Neurourology and Urodynamics, 28(4), 335-338. doi: 10.1002/nau.20628.

46. Iglesias, F., y Ocerín, J., del Molino Martín, J., Gama, E., Pérez, M., López, M., Aranguren, M. and Muñoz, J., 2000. Prevalence and Psychosocial Impact of Urinary Incontinence in Older People of a Spanish Rural Population. The Journals of Gerontology: Series A, 55(4), pp.M207-M214.

47. Stickly A, Santini Zl, and Koyanagi A (2017). Urinary incontinence, mental health and loneliness among communitydwelling older adults in Ireland. BMC Urology; 17:29. DOI 10.1186/s12894-017-0214-6

48. Fultz NH, and Herzog $A R$ (2001). Self-reported social and emotional impact of urinary incontinence. JAGS; 49:892-899.
49. Nicholson NR (2012). A review of social isolation: An important but underassessed condition in older adults. J Primary Prevent; 33:137-152. DOI 10.1007/s10935-012-0271-2.

50. Langlois F, Vu TT, Kergoat MJ, Chassé $K$, Dupuis Gilles and Bherer $L$ (2012). The multiple dimensions of frailty: physical capacity, cognition, and quality of life. International Psychogeriatrics; 24:9, 1429-1436.

51. Chong E, Chan M, Lim WS, Ding YY. Frailty Predicts Incident Urinary Incontinence Among Hospitalized Older Adults-A 1-Year Prospective Cohort Study. J Am Med Dir Assoc. 2018 May; 19(5):422-427.

52. Tannenbaum, C., Gray, M., Hoffstetter, S. and Cardozo, L. (2013). Comorbidities associated with bladder dysfunction. International Journal of Clinical Practice, 67(2), pp.105-113.

53. Norton $C$ (2013). Constipation in older patients: effects on quality of life. British Journal of NursingVol. 15, No. 4.

54. Jarvis, T., Chan, L. and Gottlieb, T., 2014. Assessment and management of lower urinary tract infection in adults. Australian Prescriber, 37(1), pp.7-9. 